\title{
Editorial: Building an excellent future
}

The past year has witnessed the dramatic growth of SCIENCE CHINA Physics, Mechanics \& Astronomy (SCPMA) under the editorial board led by former Editors-in-Chief, Professors Ding-Sheng Wang and Jie Zhang. With a raised acceptance standard for publication, SCPMA kept pace with the latest outstanding scientific advances, and organized a series of hotspot reports, including superconductors, quantum information, FAST, gravitational wave, and lunar exploration, all of which were well received by readers and authors. According to recent statistics, the English version of SCPMA has increased its 2016 impact factor to 2.237, entering the first quartile of the multidisciplinary physics category; the Chinese version of SCPMA ranks 6th among the 2008 core journals covered by the CSCI database. On behalf of the new editorial board, I would like to express sincere thanks to authors, readers and reviewers for their hard work and support.

The new editorial board is composed of 67 members, all eminent active scientists from a wide spectrum of scientific fields, research institutions and age groups. Fired with enthusiasm, they view their mission as making SCPMA the leading journal that communicates China's very best research in physics and related fields.

To strengthen our already solid foundation, the new editorial board will strive to expand the current content of SCPMA so as to include more diverse columns and report on original and edge-cutting progress to a broader audience. To attract the most interesting and innovative papers, we will continue the selection of the "Outstanding Contribution Award", which has played an important role in promoting and disseminating the research results published in SCPMA.

I would like to take this opportunity to give you my best wishes for the coming new year. I look forward to reading your submissions to SCPMA, as well as hearing any advice or feedback you might have.

\author{
Nincly Xle \\ XinCheng Xie \\ Editor-in-Chief \\ School of Physics, Peking University \\ E-mail: xcxie@pku.edu.cn \\ January 2018
}

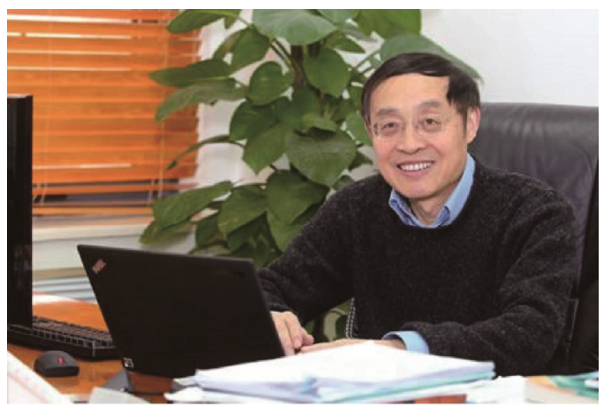

Prof. XinCheng Xie obtained his B.Sc. in physics from University of Science and Technology of China in 1982 and Ph.D. from University of Maryland in 1988. He became a faculty member in Department of Physics at Oklahoma State University in USA in 1991 and was named Regents Professor in 2004. He worked as a Chief Scientist and Director of Laboratory of Condensed Matter Theory and Computation at the Institute of Physics, Chinese Academy of Sciences in 2005-2010. Prof. Xie joined Peking University in 2010 as a Chair Professor and the Founding Director of International Center for Quantum Materials. In 2011 he was appointed Dean of School of Physics at Peking University. Prof. Xie became Division Director of Mathematical and Physical Sciences, National Natural Science Foundation of China (NSFC) in 2016. Prof. Xie was elected as a Fellow of American Physical Society in 2008 and a Member of Chinese Academy of Sciences in 2015. He has been an editorial board member of several international peer-reviewed journals, such as a Divisional Associate Editor for Condensed Matter Division of Physical Review Letters since 2013. He also serves as a member of international advisory committee of many international conferences and institutes. 